(C) [20098] IEEE. Reprinted, with permission, from [Leijia Wu, Kumbesan Sandrasegaran, and Maged Elkashlan, Tradeoff between overall throughput and throughput fairness in network controlled cell breathing algorithm, Communications, 2009. APCC 2009. 15th Asia-Pacific Conference on 8-10 Oct. 2009]. This material is posted here with permission of the IEEE. Such ermission of the IEEE does not in any way imply IEEE endorsement of any of the University of Technology, Sydney's products or services. Internal or personal use of this material is permitted.

However, permission to reprint/republish this material for advertising or promotional purposes or for creating new collective works for resale or redistribution must be obtained from the IEEE by writing to pubs-permissions@ieee.org. By choosing to view this document, you agree to all provisions of the copyright laws protecting it 


\title{
Tradeoff between Overall Throughput and Throughput Fairness in Network Controlled Cell Breathing Algorithm
}

\author{
Leijia Wu, Kumbesan Sandrasegaran, and Maged Elkashlan, Member, IEEE, \\ \{lewu,kumbes\}@eng.uts.edu.au, maged.elkashlan@csiro.au, \\ University of Technology, Sydney and Wireless Technologies Laboratory, CSIRO ICT Centre, \\ Australia
}

\begin{abstract}
The introduction of Common Radio Resource Management (CRRM) facilitates the coordination of multiple overlapped Radio Access Technologies (RATs). A Network Controlled Cell Breathing (NCCB) RAT selection algorithm is proposed in the literature for combined GSM/UMTS networks. The setting of a proper path loss threshold is a key issue in the NCCB algorithm. In this paper, the relationship among overall downlink data throughput, throughput fairness and path loss threshold is studied. It is found that in some cases, an optimum path loss threshold value can be found to achieve better performance in terms of both overall throughput and throughput fairness. However, in other cases, a tradeoff has to be made between the overall throughput and the throughput fairness.
\end{abstract}

Index Terms-common radio resource management, network controlled cell breathing, throughput fairness, integrated GSM/UMTS network

\section{INTRODUCTION}

$\mathrm{T}$ he future wireless network is expected to be a heterogeneous system, which integrates different Radio Access Technologies (RATs), such as Global System for Mobile Communications (GSM) and Universal Mobile Telecommunications System (UMTS), through a common platform. A major challenge arising from this heterogeneous network is the Radio Resource Management (RRM) strategy. Currently, RRM strategies are implemented independently in different RATs. Individually, each of these RRM strategies works well in the RAT that it is designed for. However, none of them is suitable for the heterogeneous network. In order to solve this problem, Common RRM (CRRM) strategy has been proposed in the literature to coordinate radio resource utilization across a number of RATs in an optimized way [1, 2].

The CRRM concept is based on a two-tier RRM model [3]. The RRM entity is located in the lower tier of the model. It manages radio resources within a RAT. The CRRM entity is at the upper tier of the model. It controls a number of RRM entities and can communicate with other CRRM entities. Through the information gathered from its controlling RRM entities, the CRRM entity is able to allocate a user to the most suitable RAT. Information reporting function is used by CRRM and RRM entities to communicate with each other [4]. Fig. 1 shows a basic CRRM interaction model.

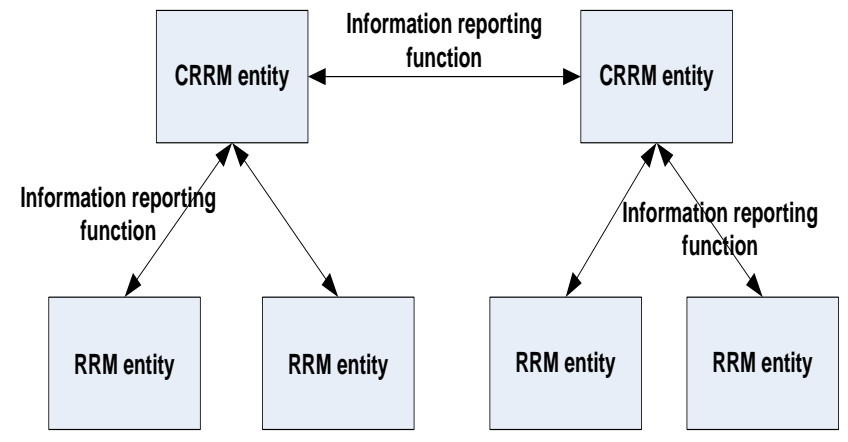

Fig. 1. CRRM interaction model

RAT selection algorithm (including initial RAT selection and vertical handover) is one of the key research areas in CRRM. A suitable RAT selection algorithm can maximize system performance by allocating users to the most suitable RAT. Different RAT selection algorithms have been studied in the literature [5]. The Network Controlled Cell Breathing (NCCB) algorithm has been proposed in the literature to coordinate radio resources in combined GSM/UMTS networks [6-8]. The setting of a proper path loss threshold is a key issue in this algorithm. The setting of a particular path loss threshold may bring higher overall throughput but cause unfairness - a small a low throughput. Or it may be the inverse case - a lower overall throughput but better throughput fairness. In this paper, the relationship among overall downlink data throughput, throughput fairness and path loss threshold is studied.

The rest of the paper is organized as follows. Section II briefly introduces the NCCB algorithm. The simulation model is described in Section III. Section IV presents the simulation results. Finally, Section V concludes this paper. 


\section{NCCB RAT SELECTION ALGORITHM}

In [6-8], a NCCB algorithm has been proposed. The NCCB concept is introduced in $[7,8]$. In FDMA/TDMA systems, there is no intra-cell interference. However, in CDMA systems, intra-cell interference exists and has significant influence on system performance. Every user transmitting data in a CDMA cell is a source of interference to all other users served in the same cell. Higher path loss requires higher transmission power and in turn generates higher interference level. A way to reduce the interference level is to control the radius of CDMA cells. The basic idea of the NCCB algorithm is to allocate high path loss users to FDMA/TDMA networks and low path loss users to CDMA networks. The path loss is measured periodically. If the path loss of a user is higher than a predefined path loss threshold, they will be directed to GSM; otherwise, they will be assigned to UMTS. If there is no enough capacity in the preferred RAT, another RAT will be selected.

As introduced in the previous section, in this algorithm, the setting of path loss threshold is a key point. In this paper, Simulations are performed to find the relationship among overall downlink data throughput, throughput fairness and path loss threshold.

\section{SIMULATION MODEL}

MATLAB is selected as the simulation tool. It is assumed that a UMTS cell and a GSM cell overlap in a square area of $\mathrm{xkm} * \mathrm{xkm}$. The border effect is alleviated by using wrap-around method. The left and right borders and top and bottom borders are connected to each other. Both GSM and UMTS Base Stations (BSs) are located at the centre of the cell. The other cells to own cell interference ratio is considered for UMTS. In order to simplify the problem, it is assumed as a fixed value during the simulation period.

Only data users are considered in our simulation. It is assumed that these data users are moving within the simulation area randomly at a speed of $3 \mathrm{~km} / \mathrm{hour}$ and continuously downloading data during the simulation period.

For GSM, it is assumed that there are three carrier frequencies in the cell. Each of them can be divided into eight time slots. So, the GSM cell has 24 physical channels. Three of them are reserved for common control purpose so that there are totally 21 channels available for data transmission. Each user will be allocated to one channel. The available throughput for each user is $14.4 \mathrm{kbps}$ [9].

For UMTS, a single FDD carrier frequency of $1950 \mathrm{MHz}$ is considered. All data users equally share the UMTS resources. If there are sufficient resources available, they can achieve a maximum throughput up to $384 \mathrm{kbps}$ in downlink. If the resources are not enough, they will be served in a lower throughput.

UMTS admission control algorithms used in this simulation are introduced in [10]. In UMTS networks, load factor is introduced to measure system load. When a UMTS network is fully loaded, its load factor is one. Because a UMTS system will be unstable if it is fully loaded (the powers of all users will reach to the maximum level in this case), a safety margin is required.
Here the maximum allowed load factor value (load factor threshold) is set to be 0.75 . In UMTS networks, the uplink and downlink load factors should be calculated separately. The uplink load factor can be calculated using (1) [10]

$$
\eta_{U L}=(1+f) \cdot \sum_{j=1}^{N} \frac{1}{1+\frac{W}{\left(E_{b} / N_{0}\right)_{j} \cdot R_{j} \cdot v_{j}}},
$$

where $\eta_{U L}$ is the uplink load factor, $f$ is the other cells to own cell interference ratio, $N$ is the number of service connections, $W$ is the WCDMA chip rate, $E_{b} / N_{0}$ is the signal energy per bit to noise spectral density ratio, $R_{j}$ is the throughput and $v_{j}$ is the activity factor of a service at physical layer. The downlink load factor is given in (2) [10]

$$
\eta_{D l}=\sum_{j=1}^{N} v_{j} \cdot \frac{\left(E_{b} / N_{0}\right)_{j}}{W / R_{j}} \cdot[(1-\bar{\alpha})+\bar{f}],
$$

where $\eta_{D L}$ is the downlink load factor, $\bar{a}$ is the average orthogonality factor in the cell and $\bar{f}$ is the average other cells to own cell interference ratio. A new service request is accepted if in uplink

$$
\text { New_ } \eta_{U L}<\eta_{U L_{-} \text {threshold }} \text {, }
$$

and the same in downlink

$$
\text { New_ } \eta_{D L}<\eta_{D L_{-} \text {threshold }} \text {, }
$$

where New $\eta_{U L}$ and New_ $\eta_{D L}$ are the uplink and downlink load factors after accepting the new user, respectively; $\eta_{U L}$-threshold and $\eta_{\text {DL-threshold }}$ are the uplink and downlink load factors thresholds, respectively.

A problem of the above UMTS admission control algorithm is that it does not consider the BS transmission power. In UMTS, the BS transmission power is limited and part of the power is reserved for common channels. The downlink transmission power calculation is given in (5) [11]

$$
P_{D L}=\frac{P_{N} \cdot \sum_{j=1}^{n} \frac{\left(E_{b} / N_{0}\right)_{j} \cdot R_{j} \cdot v_{j}}{W} \cdot L_{j}}{1-\eta_{D L}},
$$

where $P_{D L}$ is the BS transmission power for traffic channels, $P_{N}$ is the thermal noise power and $L$ is the loss between BS and UE (including path loss and penetration loss). The path loss can be calculated using (6) by assuming that the BS antenna height is $30 \mathrm{~m}$ and the mobile antenna height is $1.5 \mathrm{~m}$ [10]

$$
L_{\text {path }}=137.4+35.2 \log _{10}(d) \text {, }
$$

The penetration loss can be set to a constant value.

If a user wants to be accepted, in addition to meet the load factor requirements, it also should meet the following power requirement

$$
\text { New } P_{D L}<P_{D L_{-} \max },
$$

where New $P_{D L}$ is the BS transmission power after accepting the new user and $P_{D L_{-} \max }$ is the maximum BS transmission power allocated to traffic channels. A user is admitted only when it meets all the requirements described by (3), (4), and (7).

The downlink throughput in GSM equals to the number of users served by GSM multiplying by $14.4 \mathrm{kbps}$. According to (2), the total downlink throughput in UMTS is calculated as follows

$$
R=\frac{\eta_{D D} \cdot W}{v \cdot\left(E_{b} / N_{0}\right) \cdot[(1-\bar{\alpha})+\overline{f]}} .
$$


According to (5), the total downlink data throughput in UMTS can be calculated as

$$
R=\frac{P_{D L} \cdot W \cdot\left(1-\eta_{D L}\right)}{P_{N} \cdot\left(E_{b} / N_{0}\right) \cdot v \cdot \bar{L}},
$$

where $\overline{\boldsymbol{L}}$ is the average loss of all served users.

The downlink throughput of data users in UMTS is the minimum one calculated from (8) and (9). If the cell size is small, the average user path loss will be relatively low. The BS transmission power will then be sufficient to support users even though most of them are located at the cell edges. In this case, the throughput is limited by the load factor. However, if the cell size becomes larger, the average user path loss will be higher and the BS transmission power will be the throughput bottleneck. The detailed UMTS network parameters are summarized in Table I.

TABLE I

UMTS NETWORK PARAMETERS

\begin{tabular}{|l|l|}
\hline Parameters & \\
\hline Activity factor $\mathrm{v}_{\mathrm{j}}$ & 1 \\
\hline $\mathrm{E}_{\mathrm{b}} / \mathrm{N}_{0}$ & $5 \mathrm{~dB}$ \\
\hline Downlink throughput of user $\mathrm{R}_{\mathrm{j}}$ & Downlink: up to 384kbps \\
\hline Downlink load factor threshold & 0.75 \\
\hline WCDMA chip rate $\mathrm{W}$ & $3.84 \mathrm{Mcps}$ \\
\hline Average orthogonality $\bar{\alpha}$ & 0.5 \\
\hline Other cells to own cell interference ratio $f$ & 0.65 \\
\hline Maximum base station transmission power & $20 \mathrm{~W}$ \\
\hline Common channel power allocation & $3 \mathrm{~W}$ \\
\hline Base station antenna height & $30 \mathrm{~m}$ \\
\hline Mobile antenna height & $1.5 \mathrm{~m}$ \\
\hline Carrier frequency & $1950 \mathrm{MHz}$ \\
\hline Thermal noise power & $-108 \mathrm{dbm}$ \\
\hline
\end{tabular}

\section{SIMULATION RESULTS AND ANALYSIS}

Two simulation scenarios have been considered: cell size of $2 \mathrm{~km} * 2 \mathrm{~km}$ and cell size of $4 \mathrm{~km} * 4 \mathrm{~km}$. The path loss of every user is measured at every time interval during the simulation period. If the path loss of user $i$ is higher than the path loss threshold, it is allocated to GSM, otherwise, it is allocated to UMTS. If the GSM capacity is full, the user is allocated to UMTS even though its path loss is higher than the threshold. Both low network load (20 users served) and high network load (40 users served) cases are simulated. A user satisfaction rate is defined as to measure the throughput fairness

$$
S=n / N, \quad(10)
$$

where $n$ is the number of users whose throughput is above 32 kbps and $N$ is the total number of users served in the network. The simulation results for the scenario where cell size is $2 \mathrm{~km} * 2 \mathrm{~km}$ are shown in Fig. 2 and 3.

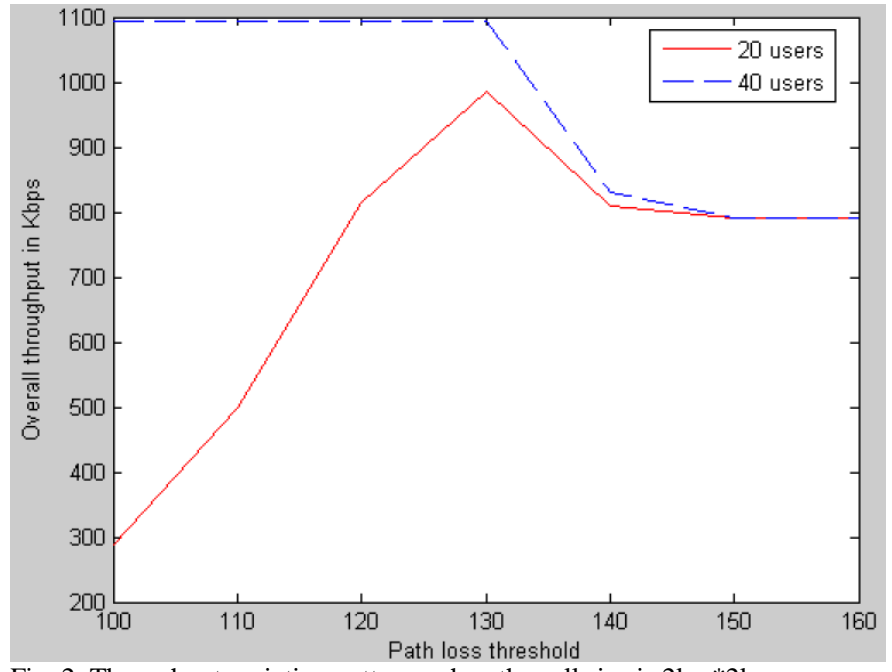

Fig. 2. Throughput variation patterns when the cell size is $2 \mathrm{~km} * 2 \mathrm{~km}$

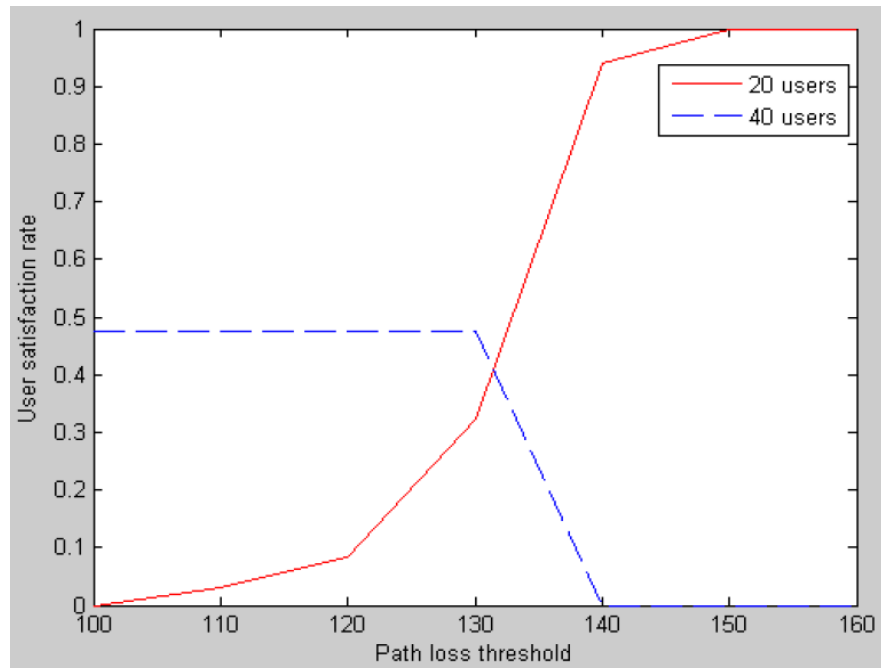

Fig. 3. User satisfaction rate variation patterns when the cell size is $2 \mathrm{~km} * 2 \mathrm{~km}$

From Fig. 2 and 3, it can be seen when the network load is low (20 users served), with the increase of path loss threshold, the overall throughput will increase to a maximum value (when the path loss threshold $=130 \mathrm{~dB}$ ) and then decrease. However, the user satisfaction rate keeps increasing. The reasons are as follows. When the path loss threshold is very low, all users are allocated to GSM, where only a relatively low throughput is available and the UMTS capacity is not utilized. This causes a very low overall throughput. And because none of the users can get a high throughput, the user satisfaction rate is also very low. However, when the path loss threshold is higher, more users will be served by UMTS, where a much higher data throughput is available. In this case, more UMTS capacity will be utilized. So the overall throughput will increase and more users can get a higher throughput, which in turn causes higher user satisfaction rate. But if we keep increasing the path loss threshold, most of or even all users will be allocated to UMTS. The UMTS capacity will reach to its maximum value and the throughput per user in UMTS will be decreased because more users are now share the same amount of resources. And because only a small number of users are assigned in GSM, most of GSM channels will be spare, which will cause a lower overall throughput. 
However, because the network load is low, even though all users are allocated to UMTS; they still can get a throughput higher than 32 kbps so that the user satisfaction rate remains increasing.

If the number of users is increased to 40 , there is no difference when the path loss threshold is 100 to $130 \mathrm{~dB}$. In these cases, all the GSM channels are occupied and the rest of users are served in UMTS. Although, in theory, more users should be allocated to GSM with the decrease of the path loss threshold, due to the limited GSM capacity, no users can be served after all the GSM channels are occupied. In this case, because of the small cell size, the overall UMTS throughput is determined by the load factor. So it is a fixed value whatever the average user path loss is. If we keep increasing the path loss threshold, both overall throughput and user satisfaction rate will decrease. The reason for overall throughput decline is the same as the case where 20 users are served. The decrease of user satisfaction rate is because a larger number of users are served in UMTS now, which causes the per user throughput less than 32 kbps.

If the cell size is enlarged to $4 \mathrm{~km} * 4 \mathrm{~km}$, the simulation results are shown in Figures 4 and 5.

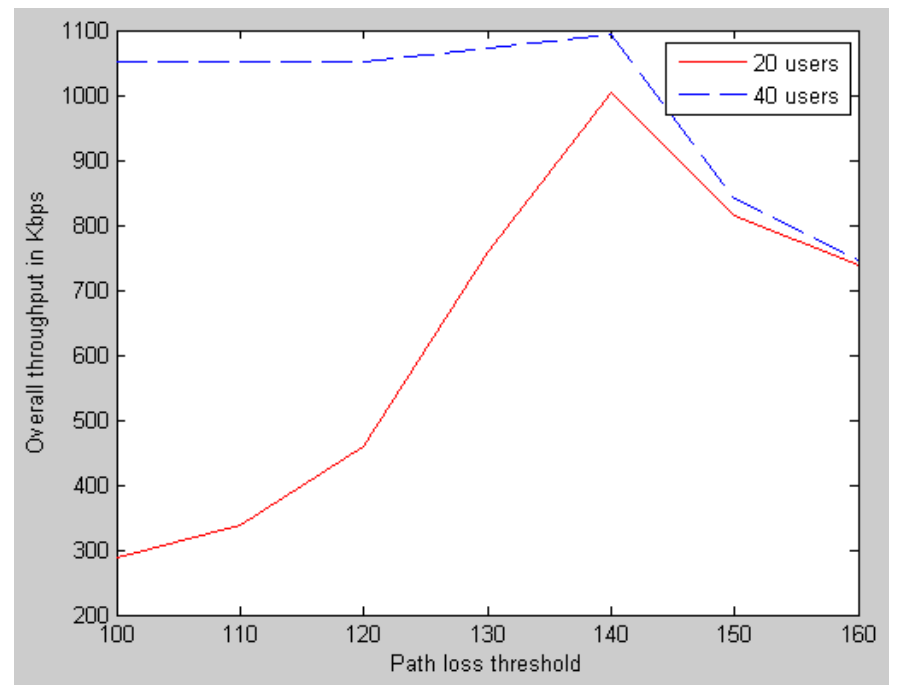

Fig. 4. Throughput variation patterns when the cell size is $4 \mathrm{~km} * 4 \mathrm{~km}$

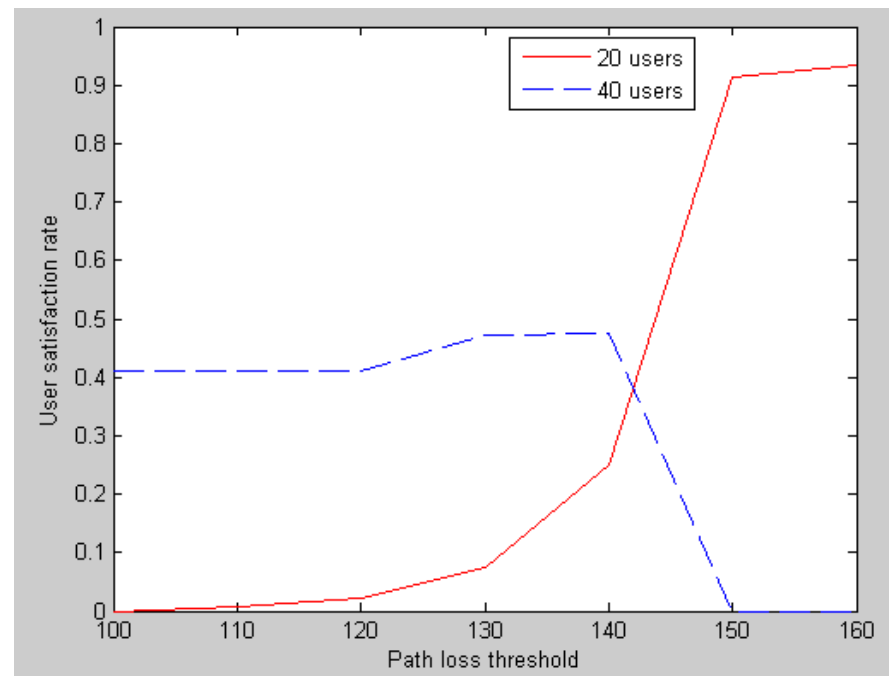

Fig. 5. User satisfaction rate variation patterns when the cell size is $4 \mathrm{~km} * 4 \mathrm{~km}$

From Figures 4 and 5, it can be seen that in both low and high network load cases, the overall throughout increases before it reaches a maximum value and then decreases, which is the same as the low network load case (20 users) when the cell size is $2 \mathrm{~km} * 2 \mathrm{~km}$. The only difference is that the maximum throughput value happens when the path loss threshold is $140 \mathrm{~dB}$. The reasons for the low network case are the same as the ones for the small cell size situation. However, in the high load case, the overall throughput variation pattern is different from the one in the small cell size case. This is because when the cell size becomes larger, the average path loss of users will increase. In this case, the throughput bottleneck is the BS transmission power rather than the load factor. With the increase of the path loss threshold, more high path loss users will be allocated to GSM so that the interference level in UMTS is reduced and higher throughput can then be achieved.

In the low network load case, the variation pattern of user satisfaction rate is the same as the one in the $2 \mathrm{~km} * 2 \mathrm{~km}$ cell size case. The reason is the same as the one for the small cell size case. In the high network load case, the user satisfaction rate will increase first because users can get a higher throughput in UMTS. However, if the path loss threshold is above $140 \mathrm{~dB}$, the user satisfaction rate decreases because there are too many users in UMTS so that the throughput per user will be reduced.

\section{CONCLUSIONS AND FUTURE WORKS}

In conclusion, with the increase of path loss threshold, the overall throughput will increase until it reaches to a maximum value (this value can be defined as $P_{L}$ ) and then it will start to decrease. The larger the cell size, the higher the value of $P_{L}$. In this simulation, the value of $P_{L}$ for the $2 \mathrm{~km} * 2 \mathrm{~km}$ cell size case is $130 \mathrm{~dB}$ while the value for the $4 \mathrm{~km} * 4 \mathrm{~km}$ cell size case is $140 \mathrm{~dB}$. When the network load is low, the user satisfaction rate will keep increasing. When the network load becomes higher, the user satisfaction rate will start to decrease when the path loss threshold is above $P_{L}$. When the network load is high, an optimum path loss threshold can be found that can achieve a best performance in terms of both overall throughput and throughput fairness. However, when the network load is low, a tradeoff is required to balance between overall throughput and throughput fairness when the path loss threshold is equal to or above $P_{L}$. The higher the path loss threshold is set, the lower the overall throughput but the better the throughput fairness.

In our future works, voice users will be included in the simulation model and the tradeoff between uplink overall throughput and throughput fairness will be studied. A multiple cell topology will be used and the value of PL will be defined more clearly.

\section{ACKNOWLEDGMENT}

This work is partly sponsored by the Commonwealth Scientific and Industrial Research Organisation (CSIRO) Information and Communication Technologies (ICT) centre. 


\section{REFERENCES}

[1] 3GPP TR v5.0.0, "Improvement of RRM across RNS and RNS/BSS (Release 5)," 2001.

[2] A. Tolli, P. Hakalin, and H. Holma, "Performance Evaluation of Common Radio Resource Management (CRRM)," in IEEE International Conference on Communications, New York, United States, 2002, pp. 3429- 3433.

[3] N. Passas, S. Paskalis, A. Kaloxylos, F. Bader, R. Narcisi, E. Tsontsis, A. S. Jahan, H. Aghvami, M. O'Droma, and I. Ganchev, "Enabling technologies for the 'always best connected' concept," Wireless Communications \& Mobile Computing vol. 6, pp. 523 5402006.

[4] J. Pterez-Romerot, O. Sal1ent, R. Agustr, P. Karlssont, A. Barbaresit, L. Wang, F. Casadevall, M. Dohler, H. Gonzfilezt, and F. Cabral-Pintot, "Common Radio Resource Management: Functional Models and Implementation Requirements," in IEEE 16th International Symposium on Personal, Indoor and Mobile Radio Communications, 2005, pp. 2067-2071.

[5] L. Wu and K. Sandrasegaran, "A Survey on Common Radio Resource Management," in The Second Australia Conference on Wireless Broadband and Ultra Wideband Communications (Auswireless07), Sydney, Australia, 2007, p. 66.

[6] J. Perez-Romero, O. Sallent, and R. Agusti, "Network Controlled Cell Breathing in Multi-Service Heterogeneous CDMA/TDMA Scenarios," in The IEEE 64th Vehicular Technology Conference (VTC-2006) Canada, 2006, pp. 1-5.

[7] J. Perez-Romero, O. Sallent, R. Agusti, N. Garcia, L. Wang, and H. Aghvami, "Network-controlled cell-breathing for capacity improvement in heterogeneous CDMA/TDMA scenarios," in Wireless Communications and Networking Conference 2006. WCNC, Las Vegas, 2006, pp. 36 - 41

[8] J. Perez-Romero, O. Salient, and R. Agusti, "A Novel Algorithm for Radio Access Technology, Selection in Heterogeneous B3G networks," in The IEEE 63rd Vehicular Technology Conference (VTC 2006-Spring), Melbourne, 2006, pp. 471 - 475

[9] J. Schiller, Mobile Communications, 2nd Edition ed. Boston: Addison-Wesley, 2003.

[10] H. Holma and A. Toskala, WCDMA for UMTS: radio access for third generation mobile communications Third edition ed.: John Wiley \& Sons, 2004.

[11] K. Sipila, Z. Honkasalo, J. Laiho-Steffens, and A. Wacker, "Estimation of Capacity and Required Transmission Power of WCDMA Downlink Based on a Downlink Pole Equation," in IEEE Proceeding of 51st. Vehicular Technology Conference Tokyo, Japan, 2000. 